\title{
COMPARISON OF DUPLEX ULTRASONOGRAPHY AND MAGNETIC RESONANCE IMAGING IN THE DETECTION OF SIGNIFICANT RENAL ARTERY STENOSIS
}

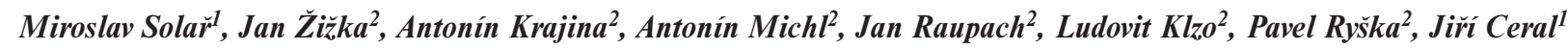

Charles University in Prague, Faculty of Medicine and University Hospital Hradec Králové, Czech Republic: Departments of Internal Medicine ${ }^{1}$ and Radiology ${ }^{2}$

Summary: Objective: The aim of our study was to evaluate duplex ultrasonography (DUS) and magnetic resonance angiography (MRA) in detection of haemodynamically significant renal artery stenosis (RAS). Methods: The study included patients with high clinical suspicion of renovascular hypertension (RVH). The imaging of renal arteries was performed by DUS, MRA and digital subtraction angiography (DSA). Significant RAS was defined as maximum systolic velocity $\geq 180$ $\mathrm{cm} / \mathrm{sec}$ (DUS) or as $60 \%$ reduction of the endoluminal arterial diameter (MRA, DSA). The results of DUS and MRA were assessed in respect to the results of DSA. Results: Arterial supply of 186 kidneys in 94 patients was evaluated. DSA revealed significant RAS in 61 kidneys evaluated. DUS was not able to examine arterial supply in 18 kidneys of 13 patients. In the detection of significant RAS, DUS was characterized by sensitivity and specificity of $85 \%$ and $84 \%$. MRA achieved satisfactory imaging quality in all but one kidney evaluated. The sensitivity and specificity of MRA in the detection of significant RAS was $93 \%$ and $93 \%$, respectively. Conclusion: In patients with high clinical probability of RVH, MRA proved to be more reliable and superior in both sensitivity and specificity to DUS in the detection of significant RAS.

Key words: Magnetic resonance imaging; Duplex sonography; Renal artery stenosis; Renovascular hypertension

\section{Introduction}

Renovascular hypertension (RVH) is one of the most common causes of secondary arterial hypertension. Renal artery stenosis (RAS) is caused by atherosclerosis in $90 \%$ of the RVH patients and fibromuscular dysplasia is found in the great majority of the remaining cases (10). As the renal revascularization might improve renal function and blood pressure control, the screening for the presence of significant renal artery stenosis (RAS) is recommended in selected patients with arterial hypertension. The main clinical clues suggesting RVH include: early or late onset of hypertension, difficult blood pressure control, coexisting atherosclerotic vascular disease and concomitant renal failure of uncertain aetiology (especially when associated with normal urinary sediment and/or precipitated by angiotensin converting enzyme inhibitors or angiotensin receptor blockers).

Non-invasive tests are currently recommended for screening of patients with suspected RVH. However, even the use of the modern and sophisticated imaging techniques such as magnetic resonance imaging or computed tomography did not yield unequivocal results in the accurate assessment of renal arteries. While several smaller studies suggest high accuracy of MRA in the detection of RAS $(8,13)$, largest performed multicentre trial did not confirm these findings (14). Therefore, the intraarterial digital subtraction angiography (DSA) still remains the golden standard for the detection of RAS (9).

In order to determine the clinical value of non-invasive approach in the screening for the significant renal artery stenosis in our centre, we decided to compare duplex ultrasonography (DUS) and magnetic resonance angiography (MRA) in patients with clinically high suspicion of RVH. While DUS was chosen because of low cost and wide availability, MRA did not expose the patients to the ionising radiation and offered decreased nephrotoxicity as well as risk of allergic reaction in comparison to computed tomography angiography or DSA.

\section{Methods}

The study included the patients with highly suspected RVH $(1,4)$ who required imaging of renal arteries. The imaging of renal arterial supply was performed by DUS, MRA and DSA in regard to the detection of significant renal artery stenosis and identification of abnormal anatomy of renal arteries. All the exams were done on daily clinical 
routine basis according to standardized protocols currently used in our institution; i.e. regardless to the study purposes. All exams were performed (or supervised) by experienced radiologists who were not aware of the results of other imaging techniques. Each of the imaging modalities was evaluated separately.

In all patients, DUS and MRA were compared in respect to DSA that was considered as golden standard technique for the detection of significant renal artery stenosis and evaluation of renal arterial anatomy. The results were analysed separately for each kidney evaluated. The sensitivities and specificities of DUS and MRA were calculated based on DSA results.

\section{Duplex ultrasonography}

DUS was performed with a $3.5 \mathrm{MHz}$ curvilinear-array transducer. The examinations were started with the patient in the supine position to visualize the origin and proximal course of the renal arteries. Both colour Doppler ultrasonography and power Doppler ultrasonography were employed in order to detect and correctly evaluate the morphology of the entire course of the renal arteries wherever possible.

Spectral Doppler waveforms were recorded for the entire courses of the renal arteries, with the colour Doppler US unit set for high velocities, to evaluate the peak systolic velocities. The Doppler angle was adjusted to the local arterial anatomy. A significant renal artery stenosis was defined by peak systolic velocity above/equal $180 \mathrm{~cm} / \mathrm{sec}$.

Tab. 1: Principal characteristics of study population.

\begin{tabular}{|l|c|}
\hline number of patients (women) & $94(41)$ \\
\hline age (years) & $60( \pm 12)$ \\
\hline age $<35$ & $5(5.3 \%)$ \\
\hline age $>75$ & $5(5.3 \%)$ \\
\hline diabetes mellitus & $31(33 \%)$ \\
\hline extrarenal signs of atherosclerosis & $50(53 \%)$ \\
\hline number of antihypertensive drugs & $3.8( \pm 1.3)$ \\
\hline systolic blood pressure $(\mathrm{mm} \mathrm{Hg})$ & $153( \pm 31)$ \\
\hline diastolic blood pressure $(\mathrm{mm} \mathrm{Hg})$ & $82( \pm 16)$ \\
\hline creatinine $>120 \mu \mathrm{mol} /$ liter & $33(35 \%)$ \\
\hline
\end{tabular}

The data are expressed as numbers of patients (percentages) or means $( \pm \mathrm{SD})$.

\section{Magnetic resonance angiography}

MR examinations were performed on a $1.5 \mathrm{~T}$ MR system with multichannel body array coil. Bolus tracking was used to monitor the arrival of contrast agent to the abdominal aorta. The MRA sequence parameters were as follows: TR $3.7 \mathrm{~ms}$; TE $1.2 \mathrm{~ms}$; flip angle $25^{\circ}$; acquisition time $18 \mathrm{sec}$; voxel size $1.1 \times 1.0$ x $1.1 \mathrm{~mm}$; centric k-space sampling; parallel acquisition techniques with acceleration factor of 2 (GRAPPA). Two experienced observers analysed MRA images. Inplane vessel diameter was measured perpendicular to the vessel axis. Significant stenosis was defined by the reduction of arterial diameter by more than $60 \%$.

\section{Digital subtraction angiography}

DSA was performed using 5-F pigtail catheter introduced via femoral artery with its tip just proximal to renal arteries. Non-ionic contrast material was injected at 20 $\mathrm{ml} / \mathrm{sec}$ for 2 seconds. Images were obtained in the anteroposterior, left anterior oblique and right anterior oblique projections. Selective renal angiography with appropriate projection adjustment was performed when the above-mentioned projections did not enable precise assessment of renal arteries. Significant stenosis was defined by the reduction of vessel diameter by more than $60 \%$.

\section{Results}

Ninety-four patients were enrolled between 2004 and 2006. Principal characteristics of study group are given in Tab. 1. All patients were examined by DUS, MRA and DSA. Arterial supply of 186 kidneys was evaluated (two of 94 patients had a solitary kidney).

\section{Digital subtraction angiography}

DSA was performed without major complications in all the patients enrolled. No problems with image interpretation were observed. Significant renal artery stenosis (>60\% in diameter) was found in 61 kidneys (32\%). The atherosclerosis was responsible for renal artery narrowing in $58 \mathrm{kid}-$ neys and the fibromuscular dysplasia causing renal artery stenosis was found in arterial system supplying 4 kidneys.

\section{Duplex ultrasonography}

Duplex ultrasonography was successfully performed in $81(86 \%)$ patients. The poor imaging quality did not enable

Tab. 2: Duplex ultrasonography and magnetic resonance angiography in the detection of significant renal artery stenosis.

\begin{tabular}{|c|c|c|c|c|c|c|c|c|c|c|}
\hline & kidneys & $\begin{array}{c}\text { kidneys } \\
\text { successfully } \\
\text { evaluated }\end{array}$ & TP & TN & FP & FN & sensitivity & specificity & PPV & NPV \\
\hline DUS & 186 & 168 & 44 & 97 & 19 & 8 & $85 \%$ & $84 \%$ & $70 \%$ & $92 \%$ \\
\hline MRA & 186 & 185 & 56 & 116 & 9 & 4 & $93 \%$ & $93 \%$ & $86 \%$ & $97 \%$ \\
\hline
\end{tabular}

The results of both methods were evaluated in regard to the results of digital substraction angiography. The data are stated as number of kidneys or percentages. DUS - duplex ultrasonography, MRA - magnetic resonance angiography, TP true positive, TN - true negative, FP - falsely positive, FN - falsely negative, PPV - positive predictive value, NPV - negative predictive value. 
proper assessment of renal arteries in 18 kidneys of 13 patients. Therefore 168 kidneys were evaluated for the presence of significant renal artery stenosis. Maximum systolic velocity $\geq 180 \mathrm{~cm} / \mathrm{sec}$, a finding defining significant stenosis, was found in 63 (37\%) examined kidneys. The comparison with DSA resulted in sensitivity and specificity of $85 \%$ and $84 \%$ (Tab. 2).

\section{Magnetic resonance angiography}

MRA achieved satisfactory imaging quality in all but one patient, where the imaging artefacts did not allow the assessment of the right renal artery. Significant stenotic lesions were observed in 65 (35\%) evaluated kidneys. Comparing MRA results with DSA resulted in the sensitivity and specificity of $93 \%$ and $93 \%$ (Tab. 2).

\section{Assessment of abnormal anatomy of renal arteries}

According to DSA, single renal artery was present in $155(83 \%)$ of examined kidneys, two renal arteries were found in 29 kidneys and three arteries were detected in two kidneys evaluated.

DUS revealed two renal arteries in nine kidneys and all of them were confirmed by DSA.

MRA described an abnormal renal artery anatomy in 34 kidneys; two renal arteries were found in 30 kidneys and three arteries were present in four kidneys. Seven supernumerary renal arteries described by MRA were not reported by DSA and, conversely, MRA did not describe two accessory arteries visible at DSA.

\section{Discussion}

We performed a clinically based comparative study to assess the value of DUS and MRA in the detection of RAS. We intended to find out whether it is effective and reasonable to perform non-invasive testing before referring the patients for DSA. The real life design of the study was chosen to describe the proper value of these techniques in a daily clinical routine. Due to the clinical selection of the patients enrolled, the prevalence of RAS in our study population was relatively high (32\%). Therefore, the observed sensitivities and specificities of DUS and MRA are related only to patients' subpopulations with high clinical probability of RVH and cannot be automatically applied to patients with moderate or low probability of RVH.

As the study procedures were actually a part of complex clinical/imaging evaluation, we could not assure that the examining radiologist was always blinded to the result of the other screening methods. However, we assume that this fact had no real impact on the observed results. The diagnostic work-up begun with DUS. Subsequent MRA was finally followed by DSA.

\section{Duplex ultrasound}

In $13 \%$ patients, poor imaging quality did not enable the assessment of renal arteries. This finding was not sur- prising as it is a well-known fact that some examinations are limited by obesity and/or bowel gas. However, the major findings concerning the DUS are lower sensitivity and negative predictive value with respect to the identification of significant renal artery stenosis. They probably reflect the inherent limitations of DUS such as difficulties in adequate imaging of the entire course of the renal artery and problems with accurate assessment of Doppler angle corrected velocity. The assessment of resistive index (15), calculated as (peak systolic velocity - end diastolic velocity)/peak systolic velocity, would have not improved the agreement between DUS and DSA. Interestingly, according to our observations, the mean value of resistive index in patients with RAS $\geq 80 \%$ was well within the physiological range, i.e. $0.59( \pm 0.11)$

Although there are some reports describing high accuracy of DUS in the detection of RAS (6), other published data support our findings $(2,3,8)$.

On the contrary, based on its wide availability, non-invasiveness and low cost, DUS can still be considered as an acceptable examination for individuals with moderate probability of RVH. However, physicians should keep in mind that negative DUS result only decreases the clinical probability of RVH and cannot definitely exclude significant RAS.

\section{Magnetic resonance imaging}

The discordance between MRA and DSA in our study resulted mainly from the evaluation of stenosis severity; the sensitivity and specificity of MRA were not optimal for the identification of significant RAS described by DSA. The observed discrepancies probably reflect the differences between both imaging techniques. Both methods can have some imperfections in the evaluation of renal artery stenosis; while MRA offers three-dimensional imaging, its image resolution is lower than that of DSA. Importantly, the reported reproducibility and interobserver agreement of DSA are not absolute.

The inaccuracies in the evaluation of the stenosis severity were also observed in other studies. Although there are some data that three-dimensional MRA leads to the overestimation of the stenosis severity $(5,7,12)$, we have observed both over- and underestimation of stenosis severity. Similar finding was recently reported by Schoenberg et al. (11).

When the MRA results were re-viewed in respect to any stenotic lesion described, MRA detected all but one significant RAS identified by DSA that would result in $99 \%$ sensitivity. This finding suggests that MRA is a clinically valuable non-invasive examination for patients with highly suspected RVH.

The great majority of RASs detected in our study were caused by atherosclerosis while the patients with fibromuscular dysplasia were sparse. Because of this reason are our results mainly applicable to the cases of atherosclerotic RAS. 


\section{Assessment of abnormal renal artery anatomy}

DUS imaging quality did not enable to depict a great proportion of accessory renal arteries and significant discrepancies were observed between MRA and DSA. This probably reflects the difficulties of both techniques to precisely describe accessory renal arteries with small diameter.

We assume that misdiagnosing of small accessory renal arteries has no real clinical impact as, based on our experience, they do not play a major role in the pathogenesis of renovascular hypertension in adults.

\section{Conclusion}

Our results suggest that in patients with high clinical probability of RVH, MRA is the examination which is reliable and superior to DUS in the detection of significant RAS.

\section{References}

1. Detection, evaluation, and treatment of renovascular hypertension. Final report Working Group on Renovascular Hypertension. Arch Intern Med 1987; 147(5):820-9.

2. De Cobelli F, Venturini M, Vanzulli A et al. Renal arterial stenosis: prospective comparison of color Doppler US and breath-hold, three-dimensional, dynamic, gadolinium-enhanced MR angiography. Radiology 2000; 214(2):373-80.

3. Kaplan-Pavlovcic S, Nadja C. Captopril renography and duplex Doppler sonography in the diagnosis of renovascular hypertension. Nephrol Dial Transplant $1998 ; 13(2): 313-7$
4. Krijnen P, van Jaarsveld BC, Steyerberg EW, Man in 't Veld AJ, Schalekamp MA, Habbema JD. A clinical prediction rule for renal artery stenosis. Ann Intern Med 1998; 129(9):705-11.

5. Mallouhi A, Schocke M, Judmaier W et al. 3D MR angiography of renal arteries: comparison of volume rendering and maximum intensity projection algorithms. Radiology 2002; 223(2):509-16.

6. Olin JW, Piedmonte MR, Young JR, DeAnna S, Grubb M, Childs MB. The Utility of Duplex Ultrasound Scanning of the Renal Arteries for Diagnosing Significant Renal Artery Stenosis. Ann Intern Med 1995; 122(11):833-8.

7. Patel ST, Mills JL Sr, Tynan-Cuisinier G, Goshima KR, Westerband A, Hughes JD. The limitations of magnetic resonance angiography in the diagnosis of renal artery stenosis: comparative analysis with conventional arteriography. J Vasc Surg 2005; 41(3):462-8

8. Rountas C, Vlychou M, Vassiou K et al. Imaging modalities for renal artery stenosis in suspected renovascular hypertension: prospective intraindividual comparison of color Doppler US, CT angiography, GD-enhanced MR angiography, and digital substraction angiography. Ren Fail 2007; 29(3):295-302.

9. Rundback JH, Sacks D, Kent KC et al. Guidelines for the Reporting of Renal Artery Revascularization in Clinical Trials. Circulation 2002; 106(12):1572-85.

10. Safian RD, Textor SC. Renal-Artery Stenosis. N Engl J Med 2001; 344(6):431-42.

11. Schoenberg SO, Rieger J, Weber CH et al. High-spatial-resolution MR angiography of renal arteries with integrated parallel acquisitions: comparison with digital subtraction angiography and US. Radiology 2005; 235(2):687-98.

12. Shetty AN, Bis KG, Kirsch M, Weintraub J, Laub G. Contrast-enhanced breathhold three-dimensional magnetic resonance angiography in the evaluation of renal arteries: optimization of technique and pitfalls. J Magn Reson Imaging 2000; 12(6):912-23.

13. Vasbinder GB, Nelemans PJ, Kessels AG, Kroon AA, de Leeuw PW, van Engelshoven JM. Diagnostic tests for renal artery stenosis in patients suspected of having renovascular hypertension: a meta-analysis. Ann Intern Med 2001; 135(6):401-11.

14. Vasbinder GB, Nelemans PJ, Kessels AG et al. Accuracy of computed tomographic angiography and magnetic resonance angiography for diagnosing renal artery stenosis. Ann Intern Med 2004; 141(9):674-82.

15. Williams GJ, Macaskill P, Chan SF. Comparative Accuracy of Renal Duplex Sonographic Parameters in the Diagnosis of Renal Artery Stenosis: Paired and Unpaired Analysis. AJR 2007; 188(3):798-811.

Received: $17 / 04 / 2009$.

Accepted in revised form: 26/10/2010.

\section{Corresponding author:}

Doc. MUDr. Miroslav Solař, Ph.D., Departement of Internal Medicine, University Hospital, Sokolská 581, Hradec Králové, 50005 Czech Republic; e-mail: solarmir@seznam.cz 